\title{
Treatment Decision-Making Capacity in Children and Adolescents Hospitalized for an Acute Mental Disorder: The Role of Cognitive Functioning and Psychiatric Symptoms
}

\author{
Gabriele Mandarelli, MD, PhD, ${ }^{1}$ Ugo Sabatello, MD,2 Elisa Lapponi, MD² Giulia Pace, MD?2 \\ Mauro Ferrara, $\mathrm{MD}^{2}$, and Stefano Ferracuti, $\mathrm{MD}^{1}$
}

\begin{abstract}
Objective: This study was conducted to assess treatment decision-making capacity (TDMC) in a child and adolescent psychiatric sample and to verify possible associations between TDMC, psychiatric symptom severity, and cognitive functioning.

Methods: Twenty-two consecutively recruited patients hospitalized for an acute mental disorder, aged 11-18 years, underwent measurement of TDMC by the MacArthur Competence Assessment Tool for Treatment (MacCAT-T). The MacCAT-T interview focused on patients' current treatment, which comprised second-generation antipsychotics (45.5\%), first-generation antipsychotics (13.6\%), antiepileptic drugs used as mood stabilizers or lithium carbonate (45.5\%), selective serotonin reuptake inhibitors (32\%), and benzodiazepines (18\%). We moreover measured cognitive functioning (Wechsler Intelligence Scale for Children III) and psychiatric symptom severity (Brief Psychiatric Rating Scale v 4.0).

Results: Patients' TDMC varied within the sample, but MacCAT-T scores were good in the sample overall, suggesting that children and adolescents with severe mental disorders could be competent to consent to treatment. The TDMC proved independent of psychiatric diagnosis while being positively associated with cognitive functioning and negatively with excitement.

Conclusion: The MacCAT-T proved feasible for measuring TDMC in a child and adolescent psychiatric sample. TDMC in minors with severe mental disorders was not necessarily impaired. These results deserve reconsidering the interplay between minors and surrogate decision-makers as concerning treatment decisions.
\end{abstract}

Keywords: informed consent, psychiatric hospitalization, treatment, mental capacity

\section{Introduction}

D ATA ON INFORMED CONSENT decision-making in child and adolescent psychiatry are scarce. Involuntary treatment and surrogate decision-making are also common in adolescent psychiatric populations, and the need for consistent legal instruments and systematic analyses of such coercive practices has been recently advocated (Jendreyschak et al. 2014).

Several studies focusing on adult psychiatric patients' treatment decision-making capacity (TDMC) found a significant role played by cognitive factors (Okai et al. 2007; Mandarelli et al. 2012). Psychiatric symptom severity, rather than diagnosis, as well as metacognition, proved to be associated with TDMC in adult psychiatric populations. Nonetheless, whether and how these factors play a role also in child and adolescent treatment decision-making is unclear. Having such information is crucial because it could provide empirical evidence to verify child and adolescent capacity to accept or refuse their own treatment as well as to usefully interact with surrogate decision-makers.

No studies specifically aimed at evaluating child and adolescent capacity to give informed consent to psychiatric treatment are available, while two studies focused on informed consent to clinical research. Among these, a methodological study (Hein et al. 2012) proposed a modified version of the MacArthur Competence Assessment Tool for Clinical Research (MacCAT-CR) for assessing capacity to give consent to clinical research in children and adolescents. A pilot study (Koelch et al. 2010) from a German research group on 12 children with ADHD or conduct disorder proved the MacCAT-CR useful for evaluating capacity to consent to a pharmacological trial. Moreover, the MacCAT-CR assessment showed that clinical judgments of capacity to consent to pharmacological research frequently were unconfirmed when using a

Departments of ${ }^{1}$ Neurology and Psychiatry and ${ }^{2}$ Pediatrics and Child Neuropsychiatry, University of Rome Sapienza, Rome, Italy.

(c) Gabriele Mandarelli et al. 2017; Published by Mary Ann Liebert, Inc. This article is available under the Creative Commons License CC-BY-NC (http://creativecommons.org/licenses/by-nc/4.0). This license permits non-commercial use, distribution and reproduction in any medium, provided the original work is properly cited. Permission only needs to be obtained for commercial use and can be done via RightsLink 
specific assessment tool such as the MacCAT-CR semistructured interview, thus suggesting the need for specific evaluations (Koelch et al. 2010).

The first research hypothesis of the present study was that the MacArthur Competence Assessment Tool for Treatment (MacCAT-T) (Grisso et al. 1997), which proved useful in adults, could also be suitable in a child and adolescent psychiatry setting. The second hypothesis was that cognitive functioning as well as psychiatric symptom severity was associated with the ability to provide informed consent to treatment.

\section{Methods}

Patients were recruited at the Child and Adolescent Neuropsychiatry Inpatient Unit of the Umberto I Policlinic Hospital in Rome. Parents or legal representative received full disclosure of the aims of the study, and if agreed, patients received a full explanation of the procedure as well. Informed consent for study participation was obtained from parents and patients. The Institutional Review Board approved the study. Diagnoses were made by the Diagnostic and Statistical Manual of Mental Disorders, 5th edition (DSM-5) (American Psychiatric Association 2013) criteria. All of the approached patients agreed to participate.

Two trained research raters assessed TDMC by the Mac-CAT-T, a semistructured interview that was developed on the basis of the main facets of treatment-related decision-making, reflecting commonly applied legal standards for competence to consent to treatment. MacCAT-T scoring followed the provided instruction manual; higher scores indicate better TDMC. Administering the MacCAT-T usually takes 20-35 minutes.

The MacCAT-T comprised four subscales: understanding, appreciating, reasoning, and expressing a choice, which are rated independently and do not concur to a total score, which is not expected. The subscales investigate understanding and retaining of the information disclosed about the disorder and treatment main features, as well as presumed associated risks and benefits, the subscale range is 0-6. Appreciating subscale assesses patient's agreement with the physician opinion about diagnosis $(2=$ agrees with all disclosed disease features or provides reasonable contrary arguments, $1=$ partially recognizes disease features, and $0=$ does not recognize suffering from disease or provide delusional arguments) and treatment (scoring similar to appreciating diagnosis, max two points), the subscale range is $0-4$. Patient's ability to provide a reasonable, logical, and coherent reason about her/his treatment choices (including treatment refusal) is evaluated by the reasoning subscale, the subscale range is $0-8$. Reasoning subscale scoring includes eliciting patients' consequential and comparative thinking and analyzing its logical consistency, as well as patient capacity to indicate possible treatment/no-treatment consequences on everyday life. Expressing a choice (rated 0-2) measures patient's ability to express a clear and nonambivalent treatment choice.

A total score for the MacCAT-T was not calculated as we focused on the four subscale scores according to the interview standard procedure. This method is in agreement with the interview structure and with a multidimensional mental capacity approach, which suggests that poor performance in just one facet/subscale may imply incapacity even in the presence of a good performance in other domains. Treatment information disclosed to the patients during MacCAT-T sessions was based on patients' current psychopharmacological prescription, which had been previously decided by the treating staff and not by the study staff. Such information was collected before the interview by discussing it with the treating staff and analyzing case notes and prescriptions.

Psychiatric symptom severity was assessed by the Brief Psychiatric Rating Scale v4.0 (BPRS). Patients' subjective symptomatology was assessed by the youth self-report (YSR). The Children's Global Assessment Scale measured global functioning. The

Table 1. Clinical and Sociodemographic Data of ACutely Hospitalized ChildRen AND Adolescents WITH PSYCHIATRIC DISORDERS

\begin{tabular}{lc}
\hline$N$ & 22 \\
Age, years, mean (SD) & $15.8(1.6)$ \\
Age, years, min/max & $11.4 / 18.0$ \\
Sex, M/F, $n(\%)$ & $15 / 7(68.2 / 31.8)$ \\
School discontinuation, $n(\%)$ & $4(18.2)$ \\
Economic family status, $n(\%)$ & \\
$\quad$ Low & $6(27.3)$ \\
$\quad$ Medium & $13(59.1)$ \\
$\quad$ High & $3(13.6)$ \\
First hospitalization, $n(\%)$ & $17(77.3)$ \\
Length of hospitalization before & $38.7(30.0) 15-111$ \\
$\quad$ assessment, mean (SD), range & $45.3(9.3) 28-64$ \\
BPRS, mean (SD), range & $45.6(12.3), 31-85$ \\
CGAS, mean (SD), range & Mean (SD), \\
\hline
\end{tabular}

MacCAT-T understanding scores

$\begin{array}{ll}<2.1 & 1(4.6) \\ 2.1-3 & 3(13.6) \\ 3.1-4 & 3(13.6) \\ 4.1-5 & 8(36.4) \\ 5.1-6 & 7(31.8)\end{array}$

$4.4(1.2), 1.9-6$

$5.1-6$

$7(31.8)$

MacCAT-T appreciating scores

$\begin{array}{lll}0 & 1(4.5) & 1.7(1.1), 0-4 \\ 1 & 2(9.1) & \\ 2 & 6(27.3) & \\ 3 & 6(27.3) & \\ 4 & 7(31.8) & \end{array}$

MacCAT-T reasoning scores

$\begin{array}{lll}1-2 & 4(18.2) & 4.6(2.1), 1-8 \\ 3-4 & 7(31.8) & \\ 5-6 & 6(27.3) & \\ 7-8 & 5(21.7) & \end{array}$

MacCAT-T expressing a choice scores

$0 \quad 2(9.1)$

$1 \quad 6(27.3)$

$1.5(0.6), 0-2$

2

$14(63.7)$

WISC-III verbal, 98.2 (22.6), 63-140

mean $(\mathrm{SD})$, range

WISC-III performance, 92.5 (17.8), 61-130

mean (SD), range

WISC-III total, $\quad 95.0$ (20.9), 61-139

mean (SD)

YSR externalizing, $\quad 63.5(14.2)$

mean (SD)

YSR internalizing, $\quad 68.8(11.3)$

mean (SD)

YSR total, mean (SD) $67.2(12.3)$

MacCAT-T, MacArthur Competence Assessment Tool for Treatment; BPRS, Brief Psychiatric Rating Scale v4.0; CGAS, Children's Global Assessment Scale; SD, standard deviation; WISC-III, Wechsler Intelligence Scale for Children-III; YSR, youth self-report. 
Table 2. Correlations Between Treatment Decision-Making Capacity, Clinical Measures, and Cognitive Functioning

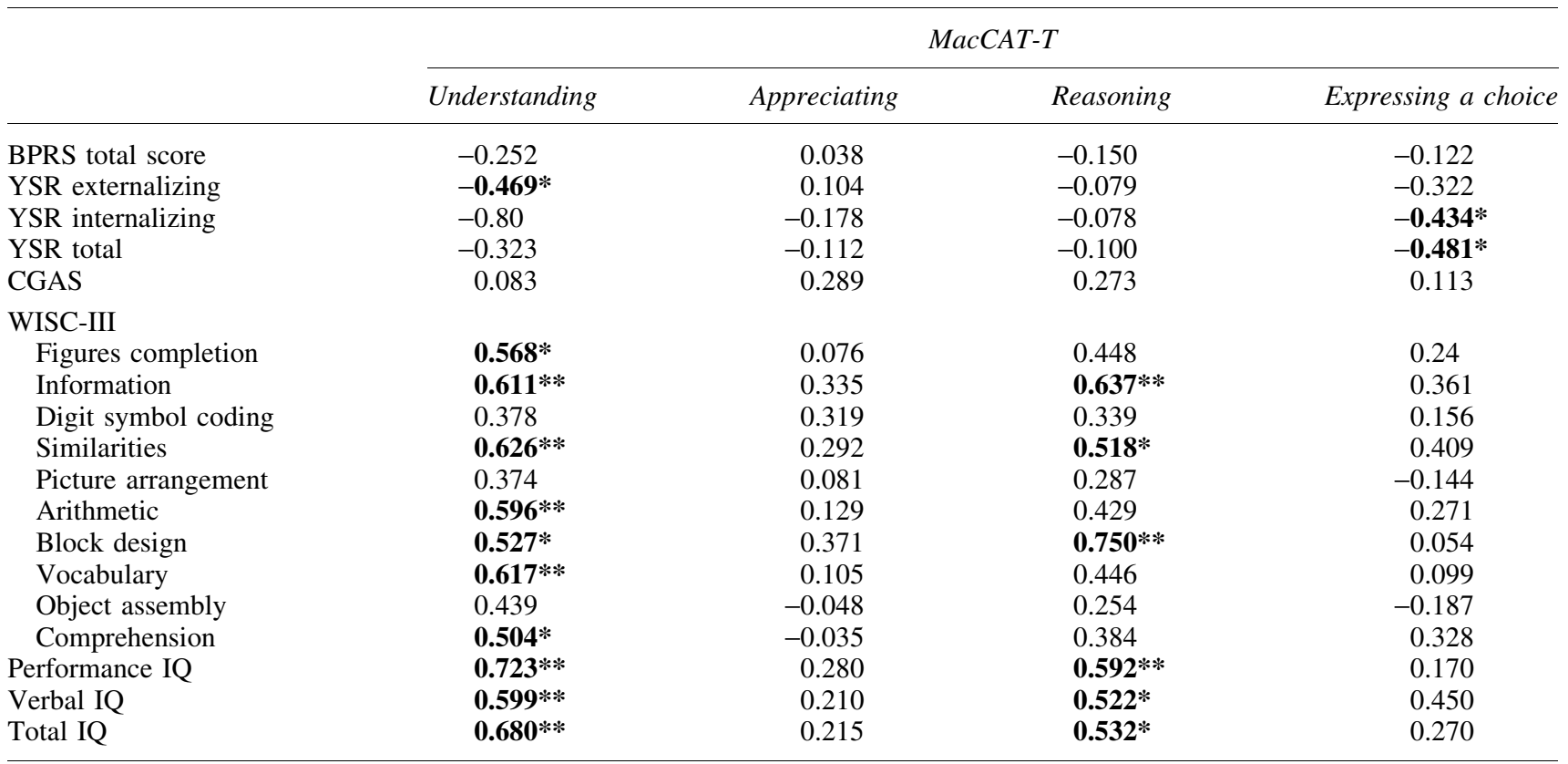

$* p<0.05 ; * *<<0.01 ; p$ values by Spearman's rho. Significant correlations are in bold.

MacCAT-T, MacArthur Competence Assessment Tool for Treatment; BPRS, Brief Psychiatric Rating Scale v4.0; CGAS, Children's Global Assessment Scale; WISC-III, Wechsler Intelligence Scale for Children-III; YSR, youth self-report.

Wechsler Intelligence Scale for Children-III (WISC-III) measured cognitive functioning.

Data were analyzed using the Statistical Package for Social Sciences, version 17.0. Correlation analysis was performed by Spearman's correlation coefficient. Kruskal-Wallis H test served to evaluate differences in MacCAT-T subscale scores among diagnostic groups (schizophrenia spectrum disorders, mood disorders, personality disorders).

\section{Results}

We recruited 22 consecutive patients aged $11-18$ years (Table 1 ) in 8 months. Thirty percent of patients suffered from bipolar and related disorders, $29 \%$ depressive disorders, $23 \%$ schizophrenia spectrum and other psychotic disorders, and $18 \%$ personality disorders. The majority of patients were at their first hospitalization. Eighty-one percent of patients received an association of more than two psychiatric medications, including second-generation antipsychotics (45.5\%), first-generation antipsychotics (13.6\%), antiepileptic drugs used as mood stabilizers or lithium carbonate $(45.5 \%)$, selective serotonin reuptake inhibitors (32\%), and benzodiazepines (18\%). There was a common use of off-label prescriptions.

All the study patients successfully completed the MacCAT-T interview. No patient completely lacked the capacity to understand and retain diagnosis and treatment information (Table 1). Mean MacCAT-T scores were also good for evaluating, reasoning, and expressing a choice. Almost $50 \%$ of the patients scored in the higher range of MacCAT-T reasoning ( $>5$ ), thus providing evidence of good capacity to rationally manipulate treatment-related information and to draw adequate conclusions about possible therapy effects in everyday life.

Nonetheless, $41 \%$ of the patients showed moderate to severe impairment in the ability of adequately recognizing their diagnosis/ symptoms as well as possible treatment implications as indicated by an appreciating score lower than 2 (Table 1). Thirty-six percent of the study patients were not able to express a clear and nonambivalent treatment choice, as indicated by having scored 0 or 1 at MacCAT-T expressing a choice (Table 1). Kruskal-Wallis H test disclosed no significant differences in capacity to consent to treatment among diagnostic groups as measured by the MacCAT-T scales.

Cognitive functioning varied widely within the study sample (median intelligence quotient $[\mathrm{IQ}]=93$, first quartile $\mathrm{IQ}=76.75$, third quartile IQ=110.25). Three patients had an IQ below 70, one patient fulfilled DSM-5 criteria for mild intellectual disability.

Spearman's rho disclosed significant correlations between cognitive measures and MacCAT-T understanding and reasoning scales (Table 2). A negative association between excitement/mania (BPRS) and understanding of treatment information emerged (Spearman's rho $=-0.498, p<0.05)$. Patients' reporting of higher emotional and behavioral problems (YSR) was associated with reduced capacity to express a clear treatment choice (Table 2).

\section{Discussion}

Our results suggest the feasibility of the MacCAT-T for measuring TDMC in child and adolescent psychiatric clinical samples. To our knowledge, this is the first study indicating the possible application of a well-established methodology for the evaluation of informed consent of such clinical population. No modifications of the published procedure were necessary.

A significant variability in TDMC emerged in the study sample, a result similar to adult psychiatric populations. However, the MacCAT-T mean values of the study sample were equal or slightly higher to those obtained by a sample of acutely hospitalized adult psychiatric inpatients (Mandarelli et al. 2014). These initial results suggest that child and adolescent psychiatric populations might 
present higher than expected TDMC. Noteworthy from a legal standpoint, all the study patients were considered incompetent as they were underage and hospitalization was decided by their parents or other surrogate decision-makers. Further studies providing external criterion validity (e.g., forensic psychiatrist judgments), including nonpsychiatric populations, will help in clarifying whether and how child and adolescent decisional capacity could be adequate also from a legal perspective. Our data suggest that severe mental disorders are not definitely associated with impaired treatment decision-making in child and adolescent psychiatry.

The ability to encode and retain information from the surrounding environment, the skills of concrete and abstract thinking, verbal competence, as well as the ability of planning and organization, are associated with the ability to rationally decide about own treatment in adolescents affected by severe mental disorders.

These data seem to confirm that cognitive domains are deeply associated with decision-making underlying treatment choices, also in children and adolescents. The correlation analyses (Table 2) showed several associations between WISC-III subscales and MacCAT-T understanding and reasoning. The lack of associations we found with two other MacCAT-T subscales (appreciating and expressing a choice) should be interpreted cautiously and needs further investigation as might be consequent to type II error in our study.

The subtest information, similarities, and vocabulary were highly correlated with the MacCAT-T understanding. This result can be interpreted in the light of a specific role played by neuropsychological functions such as discernment, confrontation, verbal memory, and visuospatial definition for this TDMC dimension. Block design correlated significantly (rho $=0.75, p<0.01$ ) with MacCAT-T reasoning, together with WISC-III information and similarities. The result seems to underline the importance of nonverbal mechanisms of conceptualization as well as planning and general organization. Executive functions, which are an acknowledged factor in adult TDMC (Mandarelli et al. 2012), are of great importance also in child and adolescent informed consent decision-making.

The lack of associations we found with MacCAT-T appreciating deserves further investigation as it seems that this TDMC facet might be specifically impaired in the study population due to noncognitive, but affective, factors.

This study has limitations; first, the small sample size impairs the possibility to generalize the study results. The single-center nature of the study requires further assessment in different clinical settings.

\section{Conclusions}

The TDMC is feasibly measurable in a child and adolescent neuropsychiatric setting with the MacCAT-T. Even though the majority of democratic countries call for surrogate treatment decision-making in minors, the initial data we provide here suggest possible good TDMC in children and adolescents affected by psychiatric disorders. Further studies should focus on TDMC in larger and nonpsychiatric samples. Our preliminary results indicate the need for enhancing the interplay between minors and surrogate decision-makers as concerning treatment decisions.

\section{Clinical Significance}

To our knowledge, this is the first study providing empirical data about TDMC in a child and adolescent clinical sample. Our results suggest that TDMC in minors might be greater than expected and deeply associated with cognitive functioning.

\section{Disclosures}

No competing financial interests exist.

\section{References}

American Psychiatric Association: Diagnostic and Statistical Manual of Mental Disorders, 5th ed. Washington, (DC), American Psychiatric Association, 2013.

Grisso T, Appelbaum PS, Hill-Fotouhi C: The MacCAT-T: A clinical tool to assess patients' capacities to make treatment decisions. Psychiatr Serv 48:1415-1419, 1997.

Hein IM, Troost PW, Lindeboom R, de Vries MC, Zwaan CM, Lindauer RJ: Assessing children's competence to consent in research by a standardized tool: A validity study. BMC Pediatr 12:156, 2012.

Jendreyschak J, Illes F, Hoffmann K, Holtmann M, Haas CR, Burchard F, Emons B, Schaub M, Armgart C, Schnieder H, Juckel G, Haussleiter IS: Voluntary versus involuntary hospital admission in child and adolescent psychiatry: A German sample. Eur Child Adolesc Psychiatry 23:151-161, 2014.

Koelch M, Prestel A, Singer H, Schulze U, Fegert JM: Report of an initial pilot study on the feasibility of using the MacArthur competence assessment tool for clinical research in children and adolescents with attention-deficit/hyperactivity disorder. J Child Adolesc Psychopharmacol 20:63-67, 2010.

Mandarelli G, Parmigiani G, Tarsitani L, Frati P, Biondi M, Ferracuti $\mathrm{S}$ : The relationship between executive functions and capacity to consent to treatment in acute psychiatric hospitalization. J Empir Res Hum Res Ethics 7:63-70, 2012.

Mandarelli G, Tarsitani L, Parmigiani G, Polselli GM, Frati P, Biondi M, Ferracuti S: Mental capacity in patients involuntarily or voluntarily receiving psychiatric treatment for an acute mental disorder. J Forensic Sci 59:1002-1007, 2014.

Okai D, Owen G, McGuire H, Singh S, Churchill R, Hotopf M: Mental capacity in psychiatric patients: Systematic review. Br J Psychiatry 191:291-297, 2007.

Address correspondence to: Gabriele Mandarelli, MD, PhD Department of Neurology and Psychiatry Viale dell'Università 30 Roma 00185 Italy

E-mail: gabriele.mandarelli@uniroma1.it 